\title{
Data Communication Using LoRa Module for Transmitting information of Flood
}

\author{
Mia Rosmiati ${ }^{1}$, Moch Fahru Rizal ${ }^{2}$, Ilma Rezza Dewa Permana ${ }^{3}$ \\ $\left\{\right.$ mia@tass.telkomuniversity.ac.id ${ }^{1}$, mfrizal@tass.telkomuniversity.ac.id ${ }^{2}$, ilmanrezza@gmail.com ${ }^{3}$ \}
}

School Of Applied Science, Universitas Telkom

\begin{abstract}
Floods are natural disasters that often occur due to overflowing rainwater in residential areas. This disaster is often caused by a blockage of water flow in the water channel. Flood disasters often occur in residential areas adjacent to rivers, such as the Bojong Soang area of Bandung, Indonesia, which every year experiences floods due to overflowing water from the Citarum River. The slow flood information received by the surrounding community from the local government has caused many moral and material losses felt by the surrounding community. Therefore we need a flood detection infrastructure that can provide information quickly to local residents. With the use of water level sensors that can detect water levels and water flow sensors that can detect the speed of water flow and be integrated with raspberry $\mathrm{Pi}$ as a data processor, the system can provide information regarding the flooding around the Citarum river. In addition, by using the LoRa module which does not require an internet connection that will be used as a medium of data communication from sensors to the data center, the condition of the Citarum River water can be easily monitored at any time. From the testing that has been done, the process of sending data from the sensor to a data center that has a distance of 400 meters has a 1-5 second delay.
\end{abstract}

Keywords: Flood Detection, Raspberry Pi, LoRa Module, Data Communication

\section{Introduction}

Flooding is a disaster that is often experienced by people who lives in Dayeuh Kolot Bandung, Indonesia every rainy season. very year water overflows and causes 10 sub-districts to be flooded, including the area of Dayeuh Kolot. In this case the flood height reached $40 \mathrm{~cm}$ to $280 \mathrm{~cm}$. This causes material losses experienced by communities around the Citarum River. The number of material losses experienced by local residents is due to the slow information received by local residents regarding the impending flood. So as to reduce the impact of the flood a system is needed that can provide early warning to the community about floods. In the study of flood monitoring and early detection using an ultrasonic sensor system, it was successful in providing early warning to the people living in the Philippine state related to the flooding that would occur via SMS via the GSM network (1). This system can provide real time response as long as it is connected to a GSM network. So the weakness of this system is the dependence on GSM networks. Whereas in the second study was a real time water condition monitoring system in Nakhon Si Thammarat, in a southern province in Thailand (2). The 
parameters observed were water level, water flow and preception level. In this study, the system built consists of three components, namely sensors, Processing / Transmission units and Databases that are on the application server. The process of sending data in real time can be done by developing a wireless sensor network through General Packet Radio Service (GPRS) as the main communication medium in sending data. While research (3) integrates sensor devices with information structures to visualize water conditions in real time. By combining LoRa and NB-IoT technology the system can transmit data at a considerable distance with low battery power usage. This system succeeded in sending data on the condition of the water flow so that the negative impacts of the floods that would occur in Korea were diminishing. In addition, the method for sending data on water conditions to prevent flooding is through the use of SMS (4). This system uses a pressure sensor to detect water level data and then the data will be read by Arduino Unio and then sent through the Global Network System For Mobile Communication (GSM) in the form of a message text to related parties.

This paper explained the process of sending data on water conditions, they are the height of water and water discharge in Citarum River. The system used water level sensor to detect The height of Citarum river, and the system used Water flow sensor DSLTD-10315036 to detect the water discharge. The process of sending data from Arduino to a server using LoRa GPS / HAT.

LoRa (Long Range) is a modulation format that has very low power. By combining RF transceiver with LoRa gateway concentrator can create a network with a large range to control millions of devices. Modulation generated using FM modulation. The core of processing produces a stable frequency value. The transmission method can also use PSK (Phase Shift Keying), FSK (Frequency Shift Keying) and others. The frequency value of the LoRa varies according to the region, if in Asia the frequency used is $433 \mathrm{MHz}$, in Europe the frequency value used is $868 \mathrm{MHz}$, while in North America the frequency used is $915 \mathrm{MHz}$ [5].The advantages of LoRa are Geolocation, Long Battery life, Long Range, High Capacity and Secured.

This paper is divided into five chapters, namely chapter 1 contains the introduction. This chapter covers the background of the research and previous studies related to this paper. In Chapter 2 discusses the method used for this study. In this section we will explain the testing techniques and retrieval of research data that are in accordance with the objectives of this paper. Whereas in Chapter 3 will discuss the result and analysis. This chapter will explain the research data along with the analysis related to the data. While in Chapter 4 will explain the conclusions of the data analysis that has been done.

\section{Research Methods}

The Flood detection system is built by integrating water levels and water flow sensors as a detector for the condition of water placed next to the Citarum rivers. The data of water condition will be processed by the Raspberry Pi as information that will be sent to the user through the LoRa Transceiver. Lora which is used has a LoRa GPS / HAT type, where this module can send sensor information and the location at once in real time. In Figure 1 below shows the water level data transmission system and river flow speed using the LoRa GPs / HAT data communication system. 


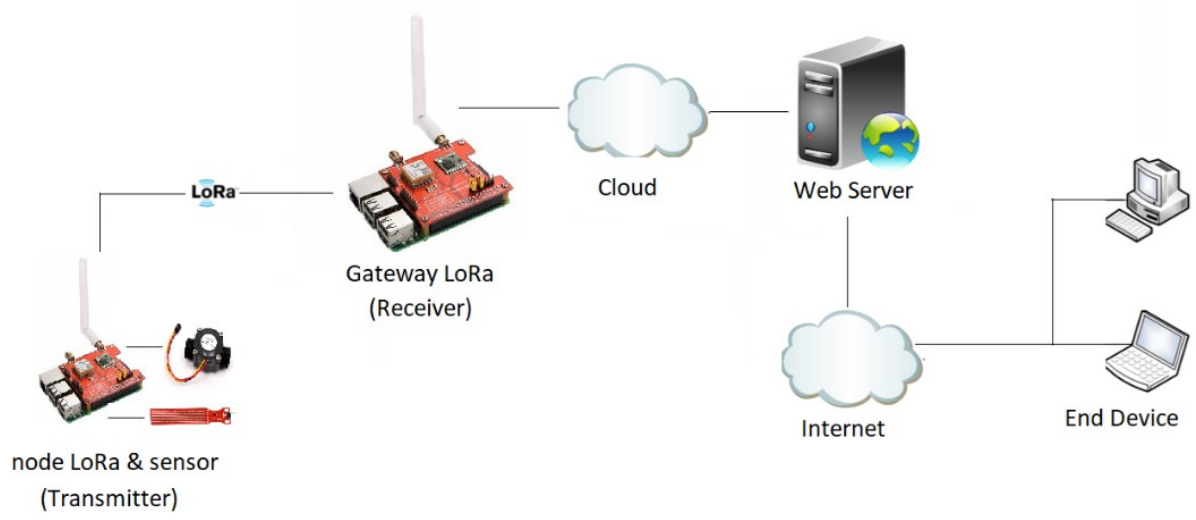

Figure 1. Data Communication fo3r Floods Detection in Citarum River.

In Figure 1 above shows the topology of the data transmission system from the sensor to the receiver. this system consists of water level sensor data and data obtained from water flow sensor measurements placed on the citarum river located in Baleendah. the data is then sent through the gateway using LoRa modulation and then sent to a server to be processed so that it can produce output on the condition of the river. While in Figure 2 shows the scheme of compilation of devices used to detect floods.

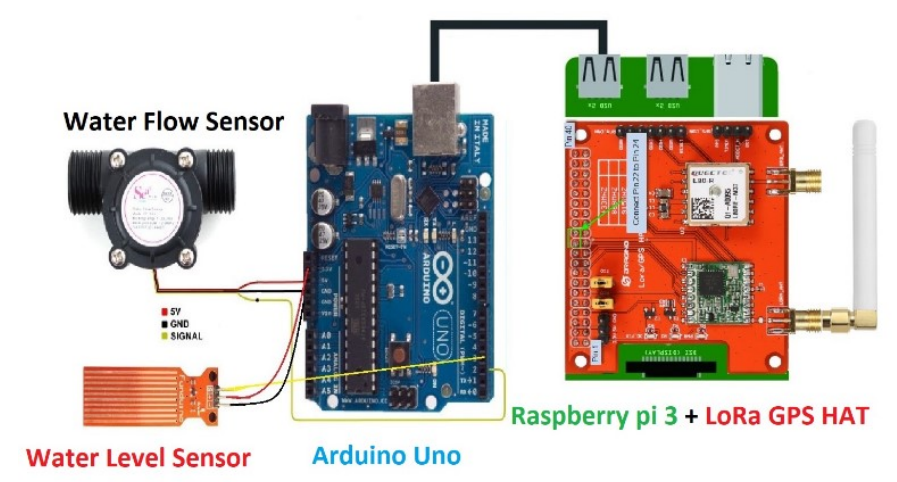

Figure 2. System of flooding detection.

In Figure 2 above shows the scheme used in the LoRa Node which is connected to Arduino as a sensor data processor then the system is connected to a Raspberry pi which has integrated to LoRa GPS HAT as a data Transmission media. In Figure 3 below shows data communication between LoRa transmitter and LoRa Receiver. 


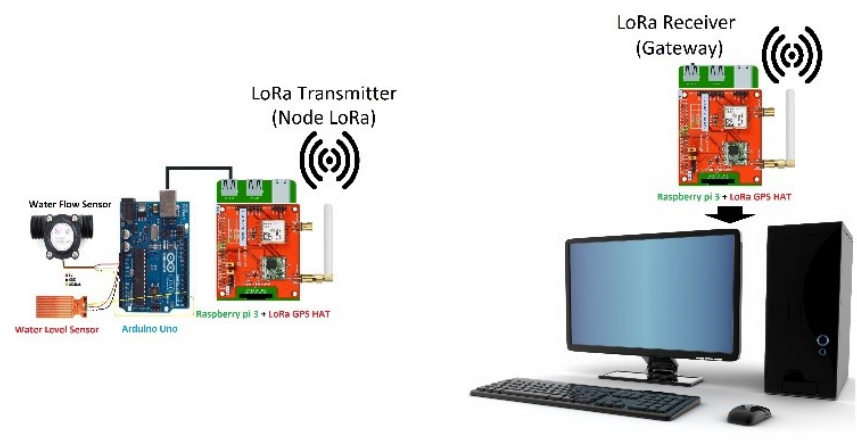

Figure 3. Data Communication using Lora Transceiver.

In Figure 3 above shows the process of sending data from the LoRa Transmitter that has information on the condition of the Citarum river to the LoRa Receiver, where at Lora receiver has function as a server that will act as the information center for the Citarum river condition.

\section{Results and Analysis}

Process of sending sensor data using the LoRa Transceiver is carried out in the Citarum River to the data center which has several obstacles such as trees and buildings made of walls. In Figure 4 shows the location of of water level sensor and water flow sensor in the Citarum River.

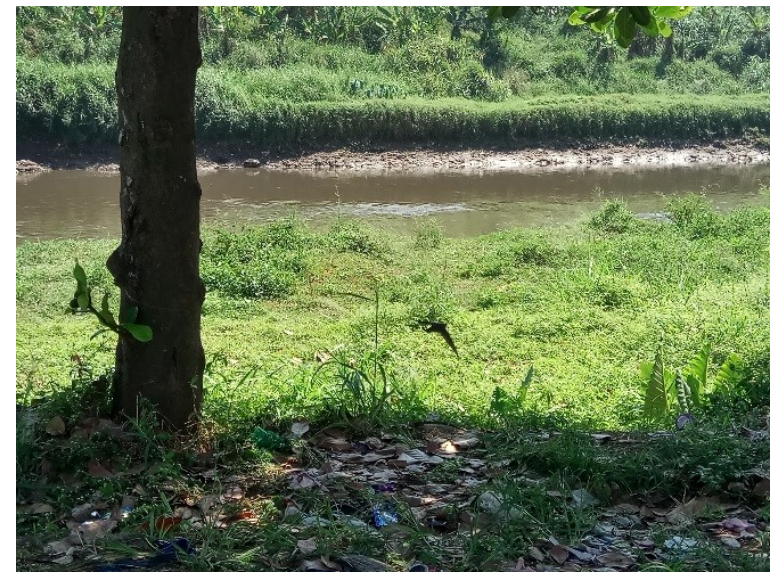

Figure 4. The Location of water flow and water level sensor.

In the Table 1 below shows the data about sensor data transmission from transmitter to receiver using variation of distance. 
Table 1. Sensor Data Transmission from transmitter to receiver.

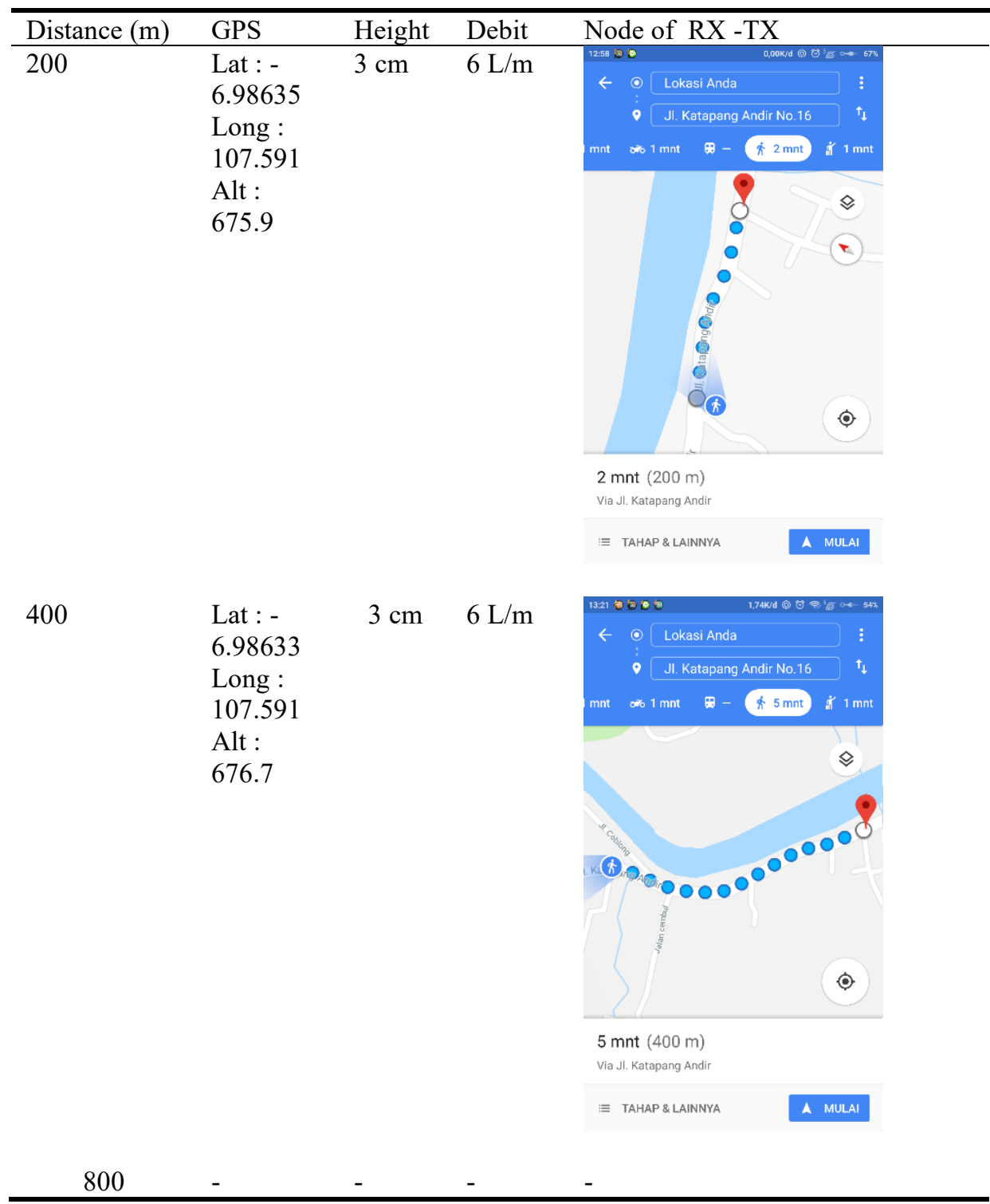

In Table 1 above, system of floods detection can send data water flow, water level and location of sensors at once from transmitter to receiver with the maximum distance of $400 \mathrm{~m}$. this is because in that location there are many trees that block the transmitter to the receiver, so the signal emitted by the LoRa Transmitter with a frequency of $915 \mathrm{MHz}$ will be scattered. 
Table 2. Time of sensor data transmission

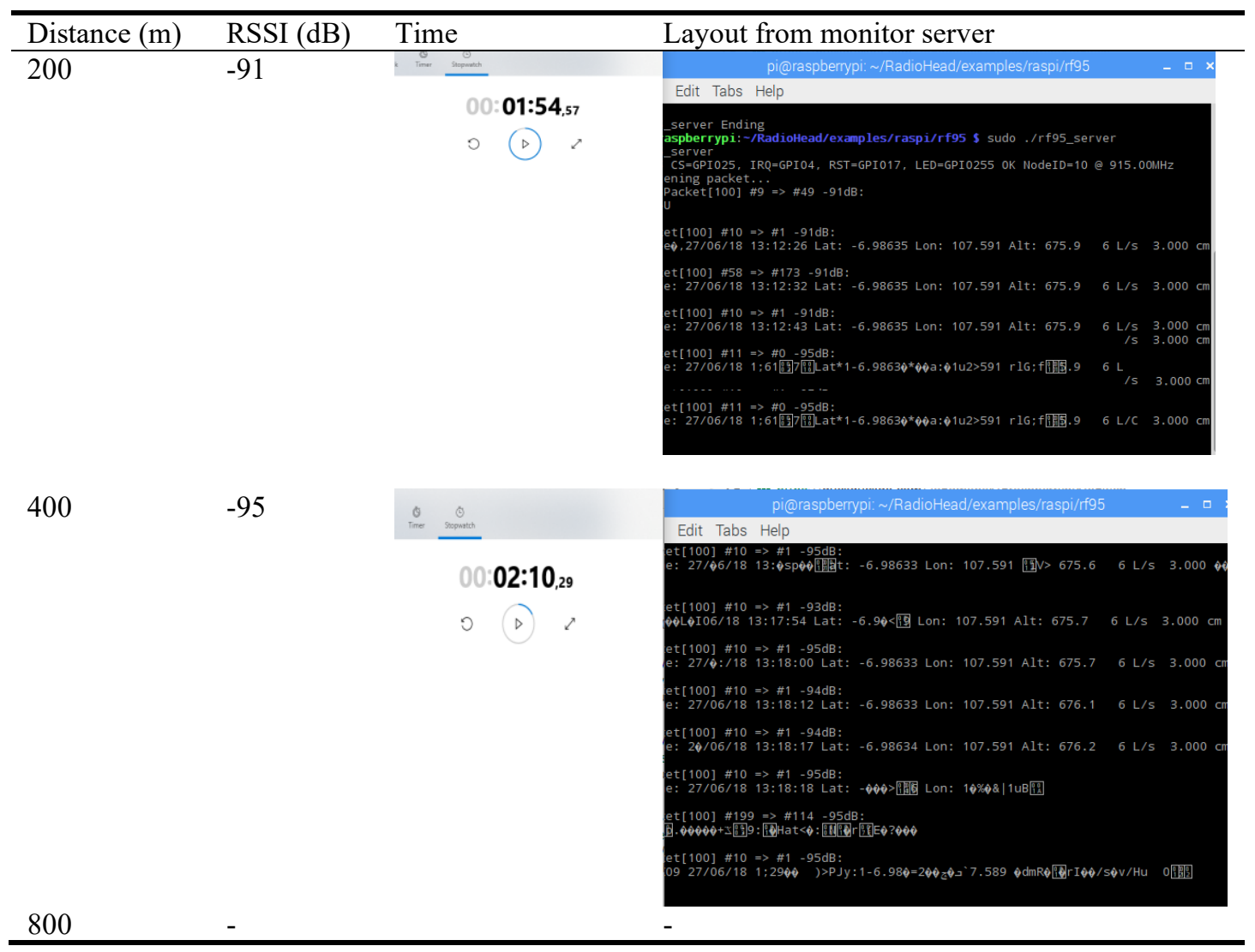

In Table 2 shows the intensity of power in the LoRa Receiver. Because of scattering, the signal in the receiver is weak. But the time is needed for sending data for transmitter to receiver about 2 second. This conclude the LoRa Transceiver can send sensor data in real time.

\section{Conclusion}

The use of LoRa transceivers can be used to send water flow sensors, water level sensors and test locations in real time even though between LoRa Transmitters and LoRa receivers there is an obstacle in the form of trees. The obstacle can cause a decrease in the intensity of the signal received by the receiver, so that from the test that has been done it can be seen that the maximum distance of the transmitter and receiver for testing data communication using LoRa is $400 \mathrm{~m}$. for further research it will be developed the integration the communication data using LoRa transceiver with web-based data monitoring and data storage automatically, so that with this system, the flood system monitoring will be easier to read by the user. 


\section{References}

[1]Natividad JG, Mendez JM. Flood Monitoring and Early Warning System Using Ultrasonic Sensor. IOP Conf Ser Mater Sci Eng. 2018;325(1).

[2] Sunkpho J, Ootamakorn C. Real-time flood monitoring and warning system. Songklanakarin J Sci Technol. 2011;33(2):227-35.

[3]Yeon S, Kang J, Lee I. A study on real-time flood monitoring system based on sensors using flood damage insurance map. Int Arch Photogramm Remote Sens Spat Inf Sci - ISPRS Arch. 2018;42(3W4):569-71.

[4]Azid S, Sharma B, Raghuwaiya K, Chand A, Prasad S, Jacquier A. SMS based flood monitoring and early warning system. ARPN J Eng Appl Sci. 2015;10(15):6387-91.

[5]Rosmiati, M., Rizal, M. F., Susanti, F., \& Alfisyahrin, G. F. (2019). Air pollution monitoring system using LoRa modul as transceiver system. Telkomnika, 